\title{
Blowing up Solution of Initial-Boundary Value Problem for a Kind of Nonlinear Evolution Equations
}

\author{
Zhongwei Zha \\ Chongqing Three Gorges University, Chongqing 404000, China \\ E-mail: sxxyzzw@163.com
}

\begin{abstract}
This dissertation is to discuss the initial-boundary value problem under the third nonlinear boundary condition for a kind of nonlinear evolution equations. To apply the maximum value theory and convex functional method£it is proved that the blowing up of solution in the definite time under some assumed conditions. The conclusion popularizes the results of references [Zhongwei Zha(2003)-Zhongwei Zha(2010)].
\end{abstract}

Keywords: Nonlinear evolution equations, Initial-boundary value problem, Blow up of solution, Convex functional method

\section{Introduction}

This paper discusses the initial-boundary value problem under the third nonlinear boundary condition for a kind of nonlinear evolution equations.

$$
\text { (I) }\left\{\begin{array}{lc}
\frac{\partial u}{\partial t}=\nabla(h(u) \nabla u)+f(x, t, u, \nabla u) & D \times(0, T) \\
h(u) \frac{\partial u}{\partial v}+u=g(x, t, u, \nabla u) & \partial D \times(0, T) \\
u(x, 0)=\varphi(x) & \bar{D}
\end{array}\right.
$$

where $D$ is a smooth boundary area in $R^{n}, x=\left(x_{1}, x_{2}, \cdots, x_{n}\right), T$ is a positive constant, $h(u)>0$ is a monotonous decreasing continuous function, $\nabla=\left(\frac{\partial}{\partial x_{1}}, \frac{\partial}{\partial x_{2}}, \cdots, \frac{\partial}{\partial x_{n}}\right)$ is a gradient operator and $\frac{\partial u}{\partial v}$ is the exterior normal derivative for $\partial D$.

The solution losing regularity and occuring blow condition in finite time study for nonlinear partial differential equations (Nonlinear Evolution Equations) related to time variable $t$ has important practical significance[Chaohao Gu(1993), Daqian Li(1989)], which are studied in [Pao C.V.(1980)-Mizoguchi N.(1997)]. However, due to the wide equation range and various characteristics of nonlinear equations, the existed results are obtained for finite solutions of specific physical problems, such as papers [Zhongwei Zha(2003)-Zhongwei Zha(2010)] are special cases of (I).

For convenience, denote $f_{\nabla u}^{\prime}=\left(\frac{\partial f}{\partial q_{1}}, \frac{\partial f}{\partial q_{2}}, \cdots, \frac{\partial f}{\partial q_{n}}\right)$, where $q_{1}=\frac{\partial u}{\partial x_{1}}, q_{2}=\frac{\partial u}{\partial x_{2}}, \cdots, q_{n}=\frac{\partial u}{\partial x_{n}} . \frac{\partial(\nabla u)}{\partial t}=\left(\frac{\partial q_{1}}{\partial t}, \frac{\partial q_{2}}{\partial t}, \cdots, \frac{\partial q_{n}}{\partial t}\right)=q_{t}^{\prime}$, and $f_{q}^{\prime} \cdot q_{t}^{\prime}=\frac{\partial f}{\partial q_{1}} \frac{\partial q_{1}}{\partial t}+\frac{\partial f}{\partial q_{2}} \frac{\partial q_{2}}{\partial t}+\cdots+\frac{\partial f}{\partial q_{n}} \frac{\partial q_{n}}{\partial t}$ denotes inner product.

For initial-boundary value problem (I), the followings are supposed:

(i) When $x \in \bar{D}, \varphi(x) \geq 0$.

(ii) $f(x, t, p, q) \in C(R) \times C^{1}[0, T) \times C^{1}(R) \times C^{1}(R), f$ and $f_{t}^{\prime}$ are non-negative and the inner product $f_{q}^{\prime} \cdot q_{t}^{\prime} \geq 0$. Denote $H(u)=\int_{o}^{u(x, t)} h(\tau) d \tau, G(p)=\int_{0}^{p} H(\zeta) d \zeta$, then there exists $\beta>1$ such that $G H f_{p}^{\prime}-\left(\beta H^{2}+G H^{\prime}\right) f \geq 0$ holds when $p \geq 0$.

(iii) $g(x, t, p, q) \in C(R) \times C^{1}[0, T) \times C^{1}(R) \times C^{1}(R)$ when $p \geq 0$ and $g \leq 0$. And when $p<0$ and $g>0$, $g_{t}^{\prime}$ and the inner product $g_{q}^{\prime} \cdot q_{t}^{\prime}$ are non-negative, while $g_{p}^{\prime}<1$.

(iv) When $x \in \bar{D}, \nabla(h(\varphi) \nabla \varphi)+f(x, 0, \varphi(x), \nabla \varphi) \geq 0$.

\section{Non-negative property of solutions for problem (I)}

In order to discuss the blowing up solutions for finite problem (I), we first prove two properties of solutions, that is

Lemma 1. If the conditions (i)-(iii) satisfy, the solution of (I) $u(x, t) \geq 0((x, t) \in \bar{D} \times[0, T))$.

Proof. If it isn't, then suppose the solution $u(x, t)$ of (I) can be taken negative number, there exists a point $P_{0}\left(x_{0}, t_{0}\right) \in$ $\bar{D} \times[0, T)$ such that $u\left(P_{0}\right)$ is the negative minimum. From the initial condition (1.3) and condition (i), $t_{0} \neq 0$. Noting the expression of $H(x, t)$ in condition (ii), it is obviously that $H(x, t)$ takes the negative minimum in $P_{0}$ too.

If $P_{0} \in D \times(0, T)$ and since $u\left(P_{0}\right)$ and $H\left(P_{0}\right)$ are the negative minimums, then $\Delta H\left(P_{0}\right) \geq 0,\left.\frac{\partial H}{\partial x_{i}}\right|_{P_{0}}=0(i=1,2, \cdots, n)$ and $\left.\frac{\partial H}{\partial t}\right|_{P_{0}} \leq 0$. But for $h(u)>0$ and from $\left.\frac{\partial H}{\partial t}\right|_{P_{0}}=\left.h(u) \frac{\partial u}{\partial t}\right|_{P_{0}},\left.\frac{\partial u}{\partial t}\right|_{P_{0}} \leq 0$ is gotten, therefore, $\left[\frac{\partial u}{\partial t}-\nabla(h(u)) \nabla u\right]_{P_{0}}=\left[\frac{\partial u}{\partial t}-\Delta H\right]_{P_{0}} \leq$ 0 , which is a contradiction that $f$ is nonnegative in condition (ii).

If $P_{0} \in \partial D \times(0, T),\left.\frac{\partial u}{\partial v}\right|_{P_{0}} \leq 0$. From the left side of the boundary condition (1.2) $\left[h(u) \frac{\partial u}{\partial v}+u\right]_{P_{0}}<0$, which is contrast 
with $g>0$ in condition (iii). So for any $(x, t) \in \bar{D} \times[0, T), u(x, t) \geq 0$. The proof is proven.

Lemma 2. If conditions (i)-(iv) satisfy, then $\frac{\partial u}{\partial t} \geq 0((x, t) \in \bar{D} \times[0, T))$.

Proof. Express the definite solution problem (I) by the following form:

$$
\left\{\begin{array}{lc}
\frac{\partial u}{\partial t}=h(u)(\nabla u)^{2}+h(u) \Delta u=f(x, t, u, \nabla u) & D \times(0, T) \\
h(u) \frac{\partial u}{\partial v}+u=g(x, t, u, \nabla u) & \partial D \times(0, T) \\
u(x, 0)=\varphi(x) & \bar{D}
\end{array}\right.
$$

Take the derivation for variable $t$, denoted by $\frac{\partial u}{\partial t}=V(x, t)$, then we obtain the initial boundary problem what $V(x, t)$ satisfies:

$$
\text { (II) }\left\{\begin{array}{cc}
\frac{\partial V}{\partial t}=h(V)(\Delta V)+2 h^{\prime}(u)(\nabla u) \cdot(\nabla V)+\left[h^{\prime \prime}(u)(\nabla u)^{2}+h^{\prime}(u)(\Delta u)+f_{u}^{\prime}\right] V \\
\quad+f_{t}^{\prime}+f_{\nabla u}^{\prime} \cdot \frac{\partial(\nabla u)}{\partial t} & D \times(0, T) \\
h(u) \frac{\partial V}{\partial v}+\left[h(u) \frac{\partial u}{\partial v}+1-g_{u}^{\prime}\right] V=g_{t}^{\prime}+g_{\nabla u}^{\prime} \cdot \frac{\partial(\nabla u)}{\partial t} & \partial D \times(0, T) \\
V(x, 0)=\nabla(h(\varphi) \nabla \varphi)+f(x, 0, \varphi, \nabla \varphi) & \bar{D}
\end{array}\right.
$$

Similar with Lemma 1 , we can prove that for any $(x, t) \in \bar{D} \times[0, T)$, the solution of mixed problem (II) $V(x, t) \geq 0$, therefore,

$$
\frac{\partial u}{\partial t} \geq 0((x, t) \in \bar{D} \times[0, T))
$$

The proof is proven.

In addition, we also need a conclusion in paper [Zhongwei Zha(1992)], that is:

Lemma 3. Suppose that $E(t)$ is a doubly differentiable function and $E(0)>0, E^{\prime}(0)<0, E^{\prime \prime} \leq 0$, then there exists $T_{0}$ such that $E\left(T_{0}\right)=0$, where $0<T_{0}<-\frac{E(0)}{E^{\prime}(0)}$.

\section{Blow-up of solution in problem (I)}

When the hypothesis conditions (i)-(iv) satisfy, the mixed problem (I) doesn't exist global smooth solution, i.e. the solution must appear blow-up within definite time, which is the following theorem:

Theorem If the conditions (i)-(iv) hold and $u(x, t)$ is the smooth solution of (I), then there exists time $T_{0}\left(0<T_{0}<+\infty\right)$, such that $\lim _{t \rightarrow T_{0}^{-}} \sup u(x, t)=\infty$.

Proof. Since $H(u)=\int_{0}^{u(x, t)} h(\tau) d \tau, H^{\prime}(u)=h(u)>0$. Noting that the definite problem (I) can be expressed the following:

$$
\text { (III) }\left\{\begin{array}{lc}
\frac{\partial u}{\partial t}=\Delta H(u)+f(x, t, u, \nabla u) & D \times(0, T) \\
h(u) \frac{\partial u}{\partial v}+u=g(x, t, u, \nabla u) & \partial D \times(0, T) \\
u(x, 0)=\varphi(x) & \bar{D}
\end{array}\right.
$$

So we only need to prove the solutions of mixed problem (III) appear blow-up within definite time under the assumed condition.

Denote

$$
\begin{aligned}
& G(u)=\int_{0}^{u} H(\zeta) d \zeta \\
& F(t)=\int_{D} \frac{1}{1+\beta}[G(u)]^{1+\beta} d x
\end{aligned}
$$

where $\beta$ is a constant in condition (ii), then $G(u)>0, F(t)<0$ and

$$
\begin{aligned}
& F(0)=\int_{D} \frac{1}{1+\beta}[G(\varphi)]^{1+\beta} d x>0 \\
& F^{\prime}(t)=\int_{D} G^{\beta} H(u) \frac{\partial u}{\partial t} d x \\
& F^{\prime}(0)=\left.\int_{D} G^{\beta} H(u) \frac{\partial u}{\partial t}\right|_{t=0}>0
\end{aligned}
$$


Take equation (3.1) into equation (6), we have

$$
F^{\prime}(t)=\int_{D} G^{\beta} H \triangle H d x+\int_{D} G^{\beta} H f d x
$$

Apply integration by parts to the first integral of the right side in equation (8) and noting the boundary condition (3.2) in (III), we have

$$
\begin{aligned}
F^{\prime}(t) & =\int_{\partial D} G^{\beta} H\left[h(u) \frac{\partial u}{\partial v}\right] d s-\int_{D} \nabla\left(G^{\beta} H\right) \cdot \nabla H d x+\int_{D} G^{\beta} H f d x \\
& =\int_{\partial D} G^{\beta} H(g-u) d s-\int_{D} \nabla\left(G^{\beta} H\right) \cdot \nabla H d x l+\int_{D} G^{\beta} H f d x
\end{aligned}
$$

where $d s$ is the area element of $\partial D$. Derivative the two sides of (9) about $t$, we have:

$$
\begin{aligned}
F^{\prime \prime}(t) & =\int_{\partial D}\left(\beta G^{\beta-1} H^{2}+G^{\beta} H^{\prime}\right)(g-u) \frac{\partial u}{\partial t} d s+\int_{\partial D} G^{\beta} H\left[g_{t}^{\prime}+g_{u}^{\prime} \frac{\partial u}{\partial t}\right. \\
& \left.+g_{\nabla u}^{\prime} \frac{\partial(\nabla u)}{\partial t}-\frac{\partial u}{\partial t}\right] d s+\int_{D}\left(\beta G^{\beta-1} H^{2}+G^{\beta} H^{\prime}\right) f \frac{\partial u}{\partial t} d x \\
& -\int_{D}\left\{\left[\beta(\beta-1) G^{\beta-2} H^{3} H^{\prime}(\nabla u)^{2}+2 \beta G^{\beta-1} G(\nabla H)^{2}\right] \frac{\partial u}{\partial t}\right. \\
& +\left[\beta G^{\beta-1} H^{2} \nabla\left(\frac{\partial u}{\partial t}\right)+\beta G^{\beta-1} H \frac{\partial H}{\partial t} \nabla u+2 G^{\beta} \nabla\left(\frac{\partial H}{\partial t}\right)\right] \cdot \nabla H \\
& \left.+\beta G^{\beta-1} H^{2}(\nabla u) \cdot \nabla\left(\frac{\partial u}{\partial t}\right)\right\} d x+\int_{D} G^{\beta} H\left[f_{t}^{\prime}+f_{u}^{\prime} \frac{\partial u}{\partial t}+f_{\nabla u}^{\prime} \cdot \frac{\partial(\nabla u)}{\partial t}\right] d x .
\end{aligned}
$$

On the other side, if derivativing the two sides of (6) about $t$ directly, we have:

$$
F^{\prime \prime}(t)=\int_{\partial D}\left(\beta G^{\beta-1} H^{2}+G^{\beta} H^{\prime}\right)\left(\frac{\partial u}{\partial t}\right)^{2} d x+\int_{D} G^{\beta} H \frac{\partial^{2} u}{\partial t^{2}} d x .
$$

We derivative the equation (3.1) about $t$, take the results into (11) and apply integration by parts, then

$$
\begin{aligned}
F^{\prime \prime}(t)= & \int_{D}\left(\beta G^{\beta-1} H^{2}+G^{\beta} H^{\prime}\right)\left(\frac{\partial u}{\partial t}\right)^{2} d x+\int_{D} G^{\beta} H\left[f_{t}^{\prime}+f_{u}^{\prime} \frac{\partial u}{\partial t}+f_{\nabla u}^{\prime} \frac{\partial(\nabla u)}{\partial t}\right] d x \\
& +\int_{\partial D} G^{\beta} H\left[g_{t}^{\prime}+g_{u}^{\prime} \frac{\partial u}{\partial t}+g_{\nabla u}^{\prime} \cdot \frac{\partial(\nabla u)}{\partial t}-\frac{\partial u}{\partial t}\right] d s \\
& -\int_{D}\left(\beta G^{\beta-1} H^{2} \nabla u+G^{\beta} \nabla H\right) \cdot \nabla\left(\frac{\partial H}{\partial t}\right) d x
\end{aligned}
$$

Double(12) and then use it to subtract(10):

$$
\begin{aligned}
F^{\prime \prime}(t)= & 2 \beta \int_{D} G^{\beta-1} H^{2}\left(\frac{\partial u}{\partial t}\right)^{2} d x+2 \int_{D} G^{\beta} H^{\prime}\left(\frac{\partial u}{\partial t}\right)^{2} d x+ \\
& \int_{D} G^{\beta} H\left[f_{t}^{\prime}+f_{\nabla u}^{\prime} \cdot \frac{\partial(\nabla u)}{\partial t}\right] d x+\int_{D} G^{\beta-1}\left[G H f_{u}^{\prime}-\left(\beta H^{2}+G H^{\prime}\right) f\right] \frac{\partial u}{\partial t} d x \\
& \int_{\partial D} G^{\beta} H\left[g_{t}^{\prime}+g_{\nabla u}^{\prime} \cdot \frac{\partial(\nabla u)}{\partial t}\right] d s+\int_{\partial D} G^{\beta} H\left(1-g_{u}^{\prime}\right) \frac{\partial u}{\partial t} d s \\
& +\int_{\partial D} G^{\beta-1}\left(\beta H^{2}+G H^{\prime}\right)(u-g) d s+\int_{D} \beta G^{\beta-1} H\left[H(\nabla H) \cdot \nabla\left(\frac{\partial u}{\partial t}\right)\right. \\
& \left.+(\nabla H) \cdot(\nabla u) \frac{\partial H}{\partial t}-H(\nabla u) \cdot \nabla\left(\frac{\partial H}{\partial t}\right)\right] d x
\end{aligned}
$$

Noting the last integral in the right side of (13) can be expressed :

$$
\int_{D} \beta G^{\beta-1} H\left[H(\nabla H) \cdot \nabla\left(\frac{\partial u}{\partial t}\right)+H^{\prime 2}(\nabla u)^{2} \frac{\partial u}{\partial t}-H H^{\prime \prime}(\nabla u)^{2} \frac{\partial u}{\partial t}\right.
$$




$$
\left.{ }^{-} H(\nabla H) \cdot \nabla\left(\frac{\partial u}{\partial t}\right)\right] d x=\int_{D} \beta G^{\beta-1} H\left(H^{\prime 2}-H H^{\prime \prime}\right)(\nabla u)^{2} \frac{\partial u}{\partial t} d x \geq 0 .
$$

In fact, since $h(u)$ is monotone decreasing positive continuous function about $u$, and from the expression of $h(u)$ and $H(u)>0, H^{\prime}(u)=h(u)>0$, while $H^{\prime \prime}(u)=h^{\prime}(u)<0$, therefore, in the above equation $H^{\prime 2}-H H^{\prime \prime}>0$.

Due to the conditions (ii)-(iii) and from the conclusions of Lemma 1 and Lemma 2, each integral in the right side of (13) is non-negative, so

$$
F^{\prime \prime}(t) \geq 2 \beta \int_{D} G^{\beta-1} H^{2}\left(\frac{\partial u}{\partial t}\right)^{2} d x .
$$

The two sides of (14) both are multiplied by $F(t)$ and apply Schwarz inequality, we have:

$$
\begin{aligned}
F^{\prime \prime}(t) F(t) & \geq \frac{2 \beta}{\beta+1} \int_{D} G^{\beta-1}\left(H \frac{\partial u}{\partial t}\right)^{2} d x \cdot \int_{D} G^{\beta+1} d x \\
& \geq \frac{2 \beta}{\beta+1}\left(\int_{D} G^{\beta} H \frac{\partial u}{\partial t} d x\right)^{2}=\frac{2 \beta}{\beta+1}\left[F^{\prime}(t)\right]^{2}
\end{aligned}
$$

Let $E(t)=[F(t)]^{-\frac{\beta-1}{\beta+1}}=[F(t)]^{-\beta_{0}}$, where $\beta_{0}=\frac{\beta-1}{\beta+1}>0$. From (5) and (7),

$$
E(0)=[F(0)]^{-\beta_{0}}>0, E^{\prime}(0)=-\beta_{0}[F(0)]^{-\left(\beta_{0}+1\right)} F^{\prime}(0)<0 .
$$

In addition, from inequality (15), $E^{\prime \prime}(t) \leq 0$. Based on Lemma 3, there exists $T_{0}\left(0<T_{0}<-\frac{E(0)}{E^{\prime}(0)}\right)$ such that $E\left(T_{0}\right)=0$, so $\lim _{t \rightarrow T_{0}^{-}} E(t)=\lim _{t \rightarrow T_{0}}[F(t)]^{-\beta_{0}}=0$, i.e. $\lim _{t \rightarrow T_{0}^{-}} F(t)=\infty$. Noting the form of $F(t)$,

$$
\lim _{t \rightarrow T_{0}^{-}} G(u(x, t))=\infty
$$

Therefore $\lim _{t \rightarrow T_{0}^{-}} \sup _{x \in \bar{D}} u(x, t)=\infty$.

In fact, if $\sup _{x \in \bar{D}} u(x, t) \leq M($ constant $)$, then

$$
G(u)=\int_{0}^{u} H(\zeta) d \zeta \leq \int_{0}^{M} H(\zeta) d \zeta \leq \int_{0}^{M}\left[\int_{0}^{u} h(\tau) d \tau\right] d \zeta \leq \int_{0}^{M}\left[\int_{0}^{M} h(\tau) d \tau\right] d \zeta \neq \infty,
$$

which is a contradiction with (16).

\section{References}

ChaoHao Gu, DaQian Li. (1993:10). Application of partial differential equations. Beijing: Higher education press.

Chipot M., Werssler F.B. (1989). Some blow up results for a nonlinear parabolic equations with a gradient term.SIAM J. Math. Anal, 20 (4): 886-907 .

DaQian Li, YunMei Chen. (1989:12). Nonlinear evolution equations. Beijing: Science press.

Gomez J.L. , Wolanski N. (1991). Blow up results and localization of blow up points for the heat equation with a nonlinear boundary condition. J. Diff. Eqs., 92 (2): 384-401.

Hailiang Zhang, Xinchun Jia. (2002). On blow-up of solution for quasilinear parabolic equations with nonlinear boundary conditions. Journal of mathematics, 22(2): 195-198.

Jucheng Deng. (1987). Blow up behavior of solutions for reaction-diffusion equation. Journal of applied mathematics, $10(4): 450-456$.

Mizoguchi N., Yanagida E. (1997). Critical exponent for the blow up of Solutions with sign Changes in a semi linear parabolic equation. Math. Ann., 307: 663-675.

Pao C.V. (1980). On the blowing up behavior of solutions for a parabolic boundary value problem. Applicable Analysis, 1(10):5-13.

YanPing Wang. (2007). Blow-up of Solution for an Initial Boundary Value Problem for a Nonlinear Hyperbolic Equation of higher order. Applied mathematics, 20 (2): 345-349.

Zhongwei Cha, Yihua Xiang. (2003). Blow-up of Solution for Mixed Problem of Quasi-Linear Parabolic Equation. Journal of sichuan university science, 40, (6): 1046-1050. 
Zhongwei Cha. (1992). Blowing-up of the Solution for an Initial Boundary Problem of Semi-linear Parabolic Equations. Applied mathematics, 5 (1): 82-87.

Zhongwei Zha. (2010). Blow up property of the solution for quasi linear parabolic equations and its application. Journal of Mathematics Research, 2(2): 51-55. 